\title{
A novel method to quantify angiogenesis in vivo using multi-photon imaging
}

\author{
R. W. Cole, ${ }^{* *}$, J. Smith* and B. J. Herron ${ }^{* * *}$ \\ * Wadsworth Center P.O. Box 509 Albany N.Y. 12201-0509; \\ ** Department of Biomedical Sciences, School of Public Health, State University of New York at \\ Albany, N.Y. 12201
}

Progress towards understanding the basic mechanisms of angiogenesis, the formation of new blood vessels from existing vasculature, have been limited by the current methods that are used to measure vessel growth. While in vivo assays provide the best environment to follow angiogenesis, inherent difficulties in obtaining reproducible data limit the power of this approach. Limitations can be attributed to several factors including variations between animals, labor intensive vessel quantification procedures and surgical methods that induce inflammatory responses that exacerbate angiogenic response. A better assay would focus on approaches that enable the measurement of vessel growth in one animal at multiple time points, while minimizing artifacts induced by experimental manipulation.

\section{Methods}

Animals All animals were handled in accordance with approved IACUC (institutional animal care and use committee) procedures. FVB/N-Tg (TIE2-lacZ)182Sato/J mice were anesthetized with isoflurane. The hind paws were then injected with $15 \mu$ l of high Concentration MATRIGEL ${ }^{\text {TM }}$ (BD Biosciences) containing $100 \mathrm{ng} / \mathrm{ml}$ VEGF165 and $300 \mathrm{ng} / \mathrm{ml}$ basic FGF (R\&D Systems Inc.). We chose to evaluate the anterior glaborous skin of the hind leg proximal to the subtalar joint as this region is easily isolated on the microscope stage and does not require hair removal prior to intravital imaging. Vital signs were monitored with the MouseOx system, Starr Life Science Corp. (Oakmount PA) throughout all procedures. Animals were given a tail vein injection of 70kD FITC conjugated dextran (Sigma Aldrich, St, Louis MO) before each imaging session.

Imaging Mice were restrained and imaged on a custom-machined microscope stage as shown in Fig. 1. The temperature of the mice was maintained by flowing $37^{\circ} \mathrm{C}$ water through tubing embedded on the stage. Mouse paws were imaged with a Leica SP5 confocal equipped for multi-photon imaging (Mai Tai laser, Spectra Physics, Tucson, AZ). The MP laser was tuned to $920 \mathrm{~nm}$ for imaging the FITC labeled dextran and $810 \mathrm{~nm}$ for imaging expressed GFP. A 20x (0.70NA) water objective lens was used to collect all $\mathrm{Z}$ stacks $(1.2 \mu \mathrm{m} / \mathrm{slice})$.

Image processing and analysis Image stacks were processed with a 3D Gaussian filter (ImagePro, Media Cybernics) to reduce noise and smooth motion introduced into individual sections by the animal's breathing and heart beat. Vessel segments were traced on composite images between branch points with ImageJ version $1.04 \mathrm{~g}$ using the neuron J plugin. Measurements were quantified by counting the total number of branch points (as a surrogate of total individual segments), total vessel length and average vessel length. 


\section{Results}

Using multi-photon intra-vital imaging, we demonstrate that it possible to repeatedly image (Z-series) the same area within a mouse paw over a period of days or even weeks Fig. 2. Our preliminary data demonstrate that highly significant $(\mathrm{p}<0.01)$ differences in branching $(35 \%$ increase in branching) and total vessel growth (28\% increase in total vessel length) can be obtained in seven days with as few as four animals. The multi-photon excitation produces the lowest photon dose in the sample of any imaging modality. This enables us to follow angiogenesis in the same animal over several days greatly minimizing sample to sample variation. Additionally by using a smaller dose of Matrigel than the conventional Matrigel plug assays [1] we can maximize the evaluation of novel angiogenic compounds when only small amounts of reagent are available.

Our ongoing work will focus on three central issues: determining the best does response and time series to measure vessel growth, development of semi automated vessel quantification procedures that minimize observational bias, and expanding our animal model to include the evaluation of more complex aspects of angiogenesis namely the incorporation of circulating endothelial progenitor cells into neovessels.

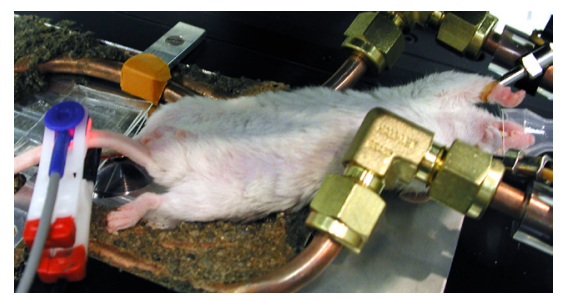

Figure 1. Demonstration of an anesthetized mouse undergoing vessel imaging.

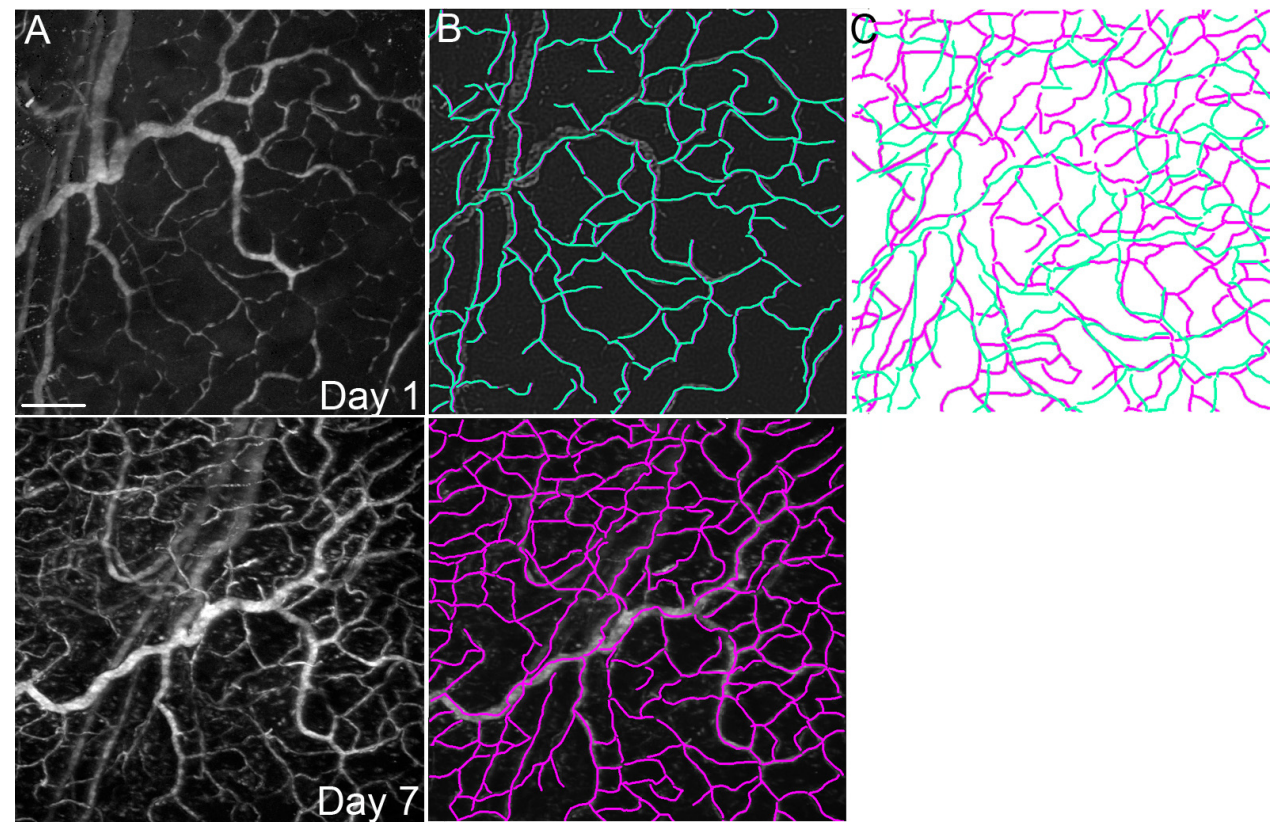

Figure 2.

(A) Representative composite image of vessels at Day 1 and Day 7 visualized with FITC Dextran.

(B) Tracings of Day1 (green) and Day 7 (purple) vessels performed in Neuron J. (C) Best-fit overlay of day 1 and day 7 tracings. $\operatorname{Bar}=100 \mu \mathrm{m}$

References

[1] R.K. Jain et al., Nat. Med. 3 (1997) 1203.

[2] P. Tiret et al., Meth. Mol. Bio. 489 (2009) 91.

[3] This work was supported by the Wadsworth Center Advanced Light Microscopy \& Image Analysis Core Facility and a grant from the NAIMS grant \# R01AR054828 \& NCRR grant \# 1S10RR023451. 